\title{
Phase Transitions in the Multicomponent Widom-Rowlinson Model and in Hard Cubes on the BCC-Lattice
}

\author{
P. Nielaba \\ Institut für Physik, Universität Mainz. D-55099 Mainz, Germany \\ J.L. Lebowitz* \\ I.H.E.S., 35, Route de Chartres, F-91440 Bures-Sur-Yvette, France
}

(September 20, 2018)

\begin{abstract}
We use Monte Carlo techniques and analytical methods to study the phase diagram of the $M$-component Widom-Rowlinson model on the bcc-lattice: there are $M$ species all with the same fugacity $z$ and a nearest neighbor hard core exclusion between unlike particles. Simulations show that for $M \geq 3$ there is a "crystal phase" for $z$ lying between $z_{c}(M)$ and $z_{d}(M)$ while for $z>z_{d}(M)$ there are $M$ demixed phases each consisting mostly of one species. For $M=2$ there is a direct second order transition from the gas phase to the demixed phase while for $M \geq 3$ the transition at $z_{d}(M)$ appears to be first order putting it in the Potts model universality class. For $M$ large, PirogovSinai theory gives $z_{d}(M) \sim M-2+2 /\left(3 M^{2}\right)+\ldots$. In the crystal phase the particles preferentially occupy one of the sublattices, independent of species, i.e. spatial symmetry but not particle symmetry is broken. For $M \rightarrow \infty$ this
\end{abstract}

${ }^{*}$ Permanent Address: Departments of Mathematics and Physics, Rutgers University, New Brunswick, NJ 08903, U.S.A. 
transition approaches that of the one component hard cube gas with fugacity $y=z M$. We find by direct simulations of such a system a transition at $y_{c} \simeq 0.71$ which is consistent with the simulation $z_{c}(M)$ for large $M$. This transition appears to be always of the Ising type. 


\section{INTRODUCTION}

The Widom- Rowlinson (WR) model, introduced in 1970 [1] as an ingeniously simple model for the study of phase transitions in continuum fluids (for an overview see Ref. [2]), continues to be, like its authors, a rich source of insights and analytical results in many (sometimes quite unexpected) areas [3] 6] of statistical mechanics. In this paper, dedicated with great pleasure to Ben Widom on the occasion of his seventieth birthday, we continue our study of a variation of the original model from two to $M$ components on a lattice: hard core exclusion between particles of different species on nearest neighbor sites.

This model was first considered by Runnels and Lebowitz [7] who proved that when the number of components $M$ is larger than some minimum $M_{0}$ then the transition from the gas phase at small values of $z$ to the demixed phase at large values of $z$ does not take place directly. Instead there is, at intermediate values of $z, z_{c}<z<z_{d}$, an ordered phase in which one of the sublattices (even or odd) is preferentially occupied, i.e. there is a crystalline (antiferromagnetically ordered) phase in which the average particle density on the even and odd sublattices, $\rho_{e}$ and $\rho_{o}$ are unequal. The average density, $\rho(I)$, of species $I=1, \ldots, M$, on each sublattice is the same for each $I$, with $\rho_{e}(I)=M^{-1} \rho_{e}$ and $\rho_{o}(I)=M^{-1} \rho_{o}$. The nature of the symmetry breaking is thus very different from that in the demixed phase at $z>z_{d}$ where $\rho_{e}=\rho_{o}=\rho$ but there exists one species, say $I^{\prime}$, for which $\rho\left(I^{\prime}\right)>M^{-1} \rho$. The

origin of this crystalline phase is purely entropic. For $z$ fixed and $M$ large "it pays" for the system "entropy wise" to occupy just one sublattice without any constraint; since there are no interactions between particles on the same sublattice there are $M$ independent choices at each site if we keep one of the sublattices empty. This more than compensates, at some $M>M_{0}$, for the "loss" of "fugacity energy" occasioned by keeping down the density in one of the sublattices.

A natural question now arises, just how big does $M_{0}$ have to be to see this ordered phase for $M \geq M_{0}$. It was shown in [7] that on the square lattice $M_{0}<27^{6}$; a ridiculously large upper bound. On the other hand a direct computation on the Bethe lattice [8,9] with 
$q$-neighbors gives $M_{0}=[q /(q-2)]^{2}$, which would suggest $M_{0} \sim 4$ for the square lattice, $M_{0} \sim 3$ for the cubic and $M_{0} \sim 2$ for the bcc lattice. Now it can be shown, using FKG inequalities, that $M_{0} \geq 3$ on any bipartite lattice [10], but beyond that we have no simple or convincing argument for finding $M_{0}$. We therefore turned to Monte Carlo simulations. This gave on the square lattice $M_{0}=7$ [8,9] which is only about twice as large as the Bethe lattice prediction. This wetted our appetite to try the bcc lattice where $q=8$. To our surprise we find here, using Monte Carlo simulations, that $M_{0}$ does indeed equal 3 , on the bcc lattice.

While we have no clue of how to find rigorously the actual value of $M_{0}$ or of $z_{c}(M)$, it was argued in [9] that for a given $z$ and $M$ large enough, the typical occupancy pattern on the lattice (ignoring the label $I$ of the particles) should be like that of a one component lattice gas with nearest neighbor hard core exclusion. For the latter system Dobrushin [11] proved the existence of a crystalline state. This implies that $z_{c}(M)$ should behave for large $M$ as $y_{c} / M$, where $y_{c}$ is the critical fugacity at which the one component hard cube gas (occupation at a site $j$ excludes occupation at all eight neighboring sites) crystalizes. The value of $y_{c}$ for the bcc lattice, obtained by Gaunt [12] using series expansion methods, is $0.77 \pm 0.05$. Using MC we obtained $y_{c}=0.71 \pm 0.01$ which is roughly consistent with Gaunt's value. Our result also agrees well with the values of $M z_{c}(M)$ for large $M$ being approximately $.72 \pm 02$ for $M=50$ and $M=100$. This provides solid evidence for the existence of a reentry phase transition in the temperature- magnetic field plane phase diagram of an Ising spin system with nearest-neighbor anti-ferromagnetic interactions on the bcc lattice [13] .

We also find, as in [9], that for large $M, z_{d}(M)$ for the crystal-demixed transition can be computed via Pirogov-Sinai theory [14] yielding,

$$
M=z_{d}+2-2 / 3 z_{d}^{2}+\cdots
$$

which matches up smoothly with our MC results, see Fig. 3. It is easy to show that there is a demixing transition for $M \geq 2$ [3, 5,14$]$, the existence of sharp interfaces between coexisting phases, for $M=2$, on the cubic lattice at large fugacity $z$ was proven in 6 . 
We next present results of our simulations and refer the reader to [9] for a more detailed description of the model and additional references.

\section{RESULTS}

Our MC simulations were carried out on a bcc lattice of size $2 \times S^{3}=2 \times 22^{3}$ with periodic boundary conditions. On an initially empty lattice we deposit particles chosen at random from the $M$ components at fugacity $z$ respecting the exclusion of different species occupying neighboring sites. We then sequentially update the lattice using a checkerboard algorithm resulting in a good vectorization. An update of a lattice site $\left(i_{1}^{(s)}, i_{2}^{(s)}, i_{3}^{(s)}\right)$ on one of the two simple cubic sublattices $s$, making up the bcc lattice, which is occupied by a particle of type $I$ ( $I=0$ indicating an empty site) is done as follows: We randomly choose a new trial particle of type $I_{t r}$, where $I_{t r}$ can have any integer value between 0 and $M$ with equal probability. $I_{t r}=0$ refers to an attempted removal of a particle $I \neq 0$ from the lattice site, which is successful, if a number $X$ randomly chosen with equal probability between 0 and 1 is smaller than the inverse fugacity $1 / z$. When this occurs $I$ gets the value 0, otherwise it remains unchanged, $I_{t r} \neq 0$ refers to an attempted deposition of a particle of type $I_{t r}$. If $I=0$ then it is successful if each of the four nearest neighbor sites is either empty or occupied by a particle of the same type $\left(I_{t r}\right)$ and $X<z$. In this case $I$ gets the value $I_{t r}$, otherwise it remains unchanged. A direct replacement attempt of a particle $I \neq 0$ surrounded by eight empty nearest neighbor sites is always successful. Typically in a simulation run after an equilibration of $2 \times 10^{5}$ Monte Carlo steps (MCS) we update the lattice $10^{5}$ times, the configuration of every tenth step is taken for the evaluation of the averages. A typical run with $10^{5}$ MCS took about 1 CPU hours on a CRAY-YMP.

Let $m\left(i_{1}^{(s)}, i_{2}^{(s)}, i_{3}^{(s)}\right)$ denote the occupancy of a site, $m\left(i_{1}^{(s)}, i_{2}^{(s)}, i_{3}^{(s)}\right)=0$ if the site $\left(i_{1}^{(s)}, i_{2}^{(s)}, i_{3}^{(s)}\right)$ is empty and $m\left(i_{1}^{(s)}, i_{2}^{(s)}, i_{3}^{(s)}\right)=1$ otherwise. As observables we took histograms $P_{L}\left(\phi_{c}\right)$ of the order parameter $\phi_{c}$ for the crystal structure and $P_{L}\left(\phi_{d}\right)$ of the order parameter $\phi_{d}$ for the demixed phase in subsystems of size $2 \times L^{3}$, 


$$
\phi_{c}=\frac{1}{2 L^{3}} \sum_{s=1}^{2} \sum_{i_{1}^{(s)}, i_{2}^{(s)}, i_{3}^{(s)}=1}^{L}\left[2 m\left(i_{1}^{(s)}, i_{2}^{(s)}, i_{3}^{(s)}\right)-1\right](-1)^{2 * s-1}
$$

and

$$
\phi_{d}=\frac{1}{2 L^{3}} \operatorname{Max}_{I} N_{L}(I)-\rho / M
$$

where $N_{L}(I)$ denotes the number of particles of type $I$ in a subsystem of size $2 \times L^{3}$ and $\rho$ is the average overall density.

\section{A. Gas-Crystal Phase Transitions}

For a given $M$ the transition activity $z_{c}$ is found by finite size scaling techniques [15, 16]. In particular the $k-t h$ moments of the order parameter distribution $P_{L}\left(\phi_{c}\right)$,

$$
<\phi_{c}^{k}>_{L}:=\int \phi_{c}^{k} P_{L}\left(\phi_{c}\right) d \phi_{c}
$$

can be evaluated in subsystems of size $2 \times L^{3}$, and from them the fourth order cumulant [16] $U_{L}$

$$
U_{L}=1-\frac{<\phi_{c}^{4}>_{L}}{3<\phi_{c}^{2}>_{L}^{2}}
$$

In a one phase region far away from a critical point the subsystem size typically can be chosen larger than the correlation length $\xi, L>>\xi$ and the order parameter distribution is to a good approximation a Gaussian centered around 0 , resulting in $U_{L} \rightarrow 0$ for $L \rightarrow \infty$. In the two phase coexistence region far away from a critical point we can again assume $L>>\xi$ and the order parameter distribution is bimodal resulting in $U_{L} \rightarrow 2 / 3$ for $L \rightarrow \infty$. Near

the critical point however we have $L<<\xi$, and using scaling arguments [16] the cumulant is a function of $L / \xi$, resulting for $\xi \rightarrow \infty$ in the same value of $U_{*}$ for all different $L$. This method allows the efficient determination of critical points by analyzing the cumulants for different values of $z$ on different length scales $L$. Applied to our model we should see, for low values of $z$, when the system is in the disordered one phase region, $U_{L^{\prime}}>U_{L}$ for $L^{\prime}<L$. 
Increasing $z$, we obtain, for large enough $M$, a crystal phase with $U_{L^{\prime}}<U_{L}$ for $L^{\prime}<L$. Near $z_{c}$ we expect $U_{L^{\prime}} \approx U_{L}$ for $L^{\prime} \neq L$. This method for locating the transition fugacities was used in our previous studies [8,9] on the square lattice as well.

For $M=3,4,5,10$ we obtain in this way values of $z_{c}=0.525 \pm 0.025,0.3,0.21,0.085 \pm 0.01$ respectively, for $M=15,17,19, z_{c}=0.055,0.047,0.04 \pm 0.001$ and for $M=1000, z_{c}=$ $0.000725 \pm 0.000025$. Cumulants for the gas-crystal transition of the hard diamond and the $M=3$-component system are shown in Figs. 1 and 2, respectively. The transition points are presented in Fig. 3 together with the asymptotic expression $M z_{c}=0.71$. This corresponds to the value of $y_{c}$ on the bcc lattice $y_{c}=0.71 \pm 0.01$ which we obtained using MC techniques. We also show in Fig. 3 a purely empirical fit of the $1 / M^{2}$-corrections for $z_{c}$, $z_{c}=0.71 / M+C / M^{2}$, to the MC data, with a value of $C=2 \pm 0.5$. The minimum number of components required for the existence of the crystal phase on the bcc lattice is $M=3$. In Fig. 4 we show the approach of the critical fugacity $M z_{c}$ to the limit of the hard diamond system.

\section{B. The Demixing Transition}

For $M=2$ we obtain a direct transition from the gas phase to the demixed phase at a transition fugacity of $z=0.55 \pm 0.02$; the transition is second order with Ising exponents as on a simple cubic lattice studied by Dickman et al. [17].

For $M>2$ we observe a direct first order transition from the crystal to the demixed phase. This transition was analyzed by studying the order parameter distribution $P_{L}\left(\phi_{d}\right)$. In the simulations we find a hysteresis region around $z_{d}$, going approximately between two values of $z$, say $z_{1}$ and $z_{2}$, when increasing and decreasing the fugacity. In cases of a small hysteresis region with extent of less than $\left|z_{1}-z_{2}\right|<0.1, z_{d}$ was taken as the average of this region, $z_{d}=\left(z_{1}+z_{2}\right) / 2$. In cases of a larger hysteresis region we located the transition fugacity $z_{d}$ by finding the relative stability of one of the two phases during a simulation starting from configurations with both phases present in parallel slices extending over the 
length of the simulation box, as described in [9]. The resulting phase transition values of $z_{d}$ are shown in Fig. 1. We note that with increasing number of components, the transition fugacities approach the exact asymptotic line $M=z+2-2 / 3 z^{2}+\cdots$.

\section{ACKNOWLEDGMENTS}

We thank A. Mazel for very helpful discussions. P.N. acknowledges support from the Deutsche Forschungsgemeinschaft (Heisenberg foundation), the computations were carried out at the CRAY-YMP of the RHRK Kaiserslautern and the CRAY-T90 of the HLRZ Jülich. J.L.L. was supported by NSF Grant DMR 95-23266 and DIMACS and its supporting agencies, the NSF under contract STC-91-19999 and the N.J. Commission on Science and Technology. 


\section{REFERENCES}

[1] B. Widom and J.S. Rowlinson, J. Chem. Phys. 52, 1670 (1970).

[2] J.S. Rowlinson and B. Widom, Molecular Theory of Capillarity, Clarendon Press, Oxford (1982), chapter 5; J. S. Rowlinson, Adv. Chem. Phys. 41, 1 (1980).

[3] J. Bricmont, K. Kuroda and J.L. Lebowitz, Z. Wahrscheinlichkeitstheor. Verw. Geb. 67, $121(1984)$.

[4] D. Ruelle, Phys. Rev. Lett. 27, 1040 (1971).

[5] L. Chayes, R. Kotecky, S.B. Shlosman, Commun. Math. Phys. 171, 203 (1995).

[6] J.Bricmont, J.L.Lebowitz, C.E. Pfister and E.Olivieri, Comm. Math. Phys., 66, 1 (1979)

[7] L.K. Runnels, J.L. Lebowitz, J. Math. Phys. 15, 1712 (1974).

[8] J.L. Lebowitz, P. Nielaba, L. Samaj, in: Computer Simulation Studies in Condensed Matter Physics VIII, D.P. Landau, K.K. Mon, H.B. Schüttler (eds.), Springer, Berin (1995), pp. 129.

[9] J.L. Lebowitz, A. Mazel, P. Nielaba, L. Samaj, Phys. Rev. E52, 5985 (1995).

[10] J.L. Lebowitz, A. Martin-Lof, Commun. Math. Phys. 25, 276 (1972); M. Cassandro, G. Gallavotti, J.L. Lebowitz, J.L. Monroe, Commun. Math. Phys. 32, 153 (1973).

[11] R.L. Dobrushin, Funct. Anal. Appl. 2, 302 (1968).

[12] D.S.Gaunt, J. Chem. Phys. 46, 3237 (1967).

[13] D.P. Landau, Phys. Rev. B16, 4164 (1977).

[14] S.A.Pirogov and Ya.G.Sinai, Phase Diagrams of the Classical Lattice Systems, Theor. Math. Phys. 25, 1185-1192 (1975) and 26 39-49 (1976).

[15] Finite Size Scaling and Numerical Simulation, edited by V. Privman (World Scientific, 
Singapore, 1990); M.N. Barber, in Phase Transitions and Critical Phenomena 8, edited by C. Domb and J.L. Lebowitz (Academic, London, 1983).

[16] K. Binder, Z. Phys. B 43, 119 (1981).

[17] R. Dickman and G. Stell, J. Chem. Phys. 102, 8674 (1995). 


\section{FIGURES}

FIG. 1. Cumulants versus $z$ for the gas-crystal transition for $M=3$. Symbols indicate different subsystem sizes, lines are for visual help.

FIG. 2. Cumulants versus $z$ for the gas-crystal transition for the hard diamond system on the bcc lattice. Symbols indicate different subsystem sizes, lines are for visual help.

FIG. 3. Phase diagram in the $M-z$ plane for a bcc lattice (MC). Full lines: Asymptotic lines for the phase transitions in the high fugacity region, $M=z+2-2 / 3 z^{2}$, and for the transition in the low fugacity region, $M z=0.71 \pm 0.01$, dashed line: transition line in the low fugacity region by fit of the $1 / M^{2}$ corrections for $z_{c}$ to the MC data, $z_{c}=0.71 / M+C / M^{2}$, with $C=2 \pm 0.5$. Symbols for MC: Transition points from the gas phase to the crystal phase (circles), from the gas to the demixed phase (squares) and from the crystal to the demixed phase (triangles).

FIG. 4. Critical fugacities $M z_{c}$ versus $1 / M$ for the gas-crystal transition. The open symbols are results of the present Monte Carlo simulations, The black symbol (with error bars) on the $y$-axis indicates the critical fugacity of the hard diamond system from series expansions [12], the open symbol on the $y$-axis the critical fugacity from our MC simulations. Lines are for visual help. 


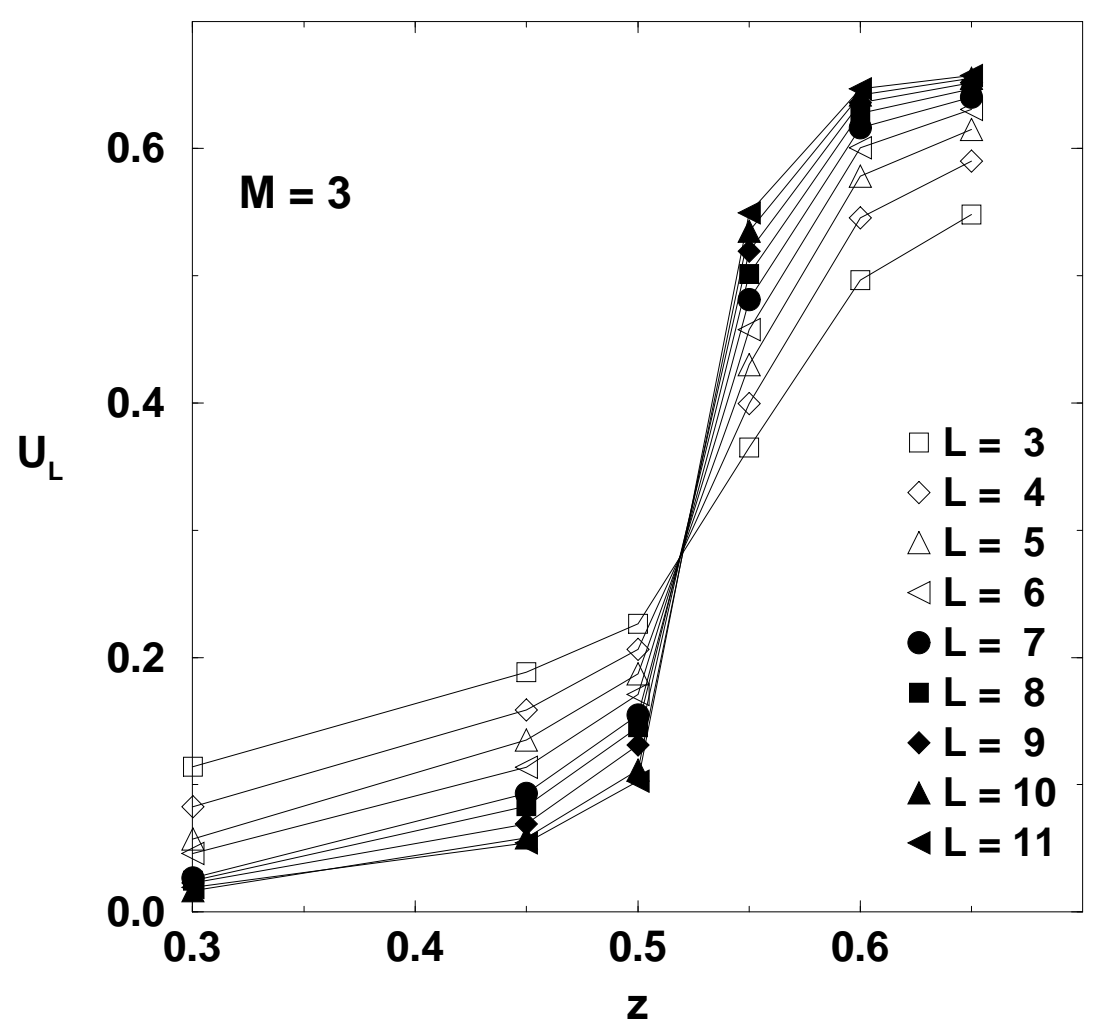

Figure 1 (Nielaba and Lebowitz)

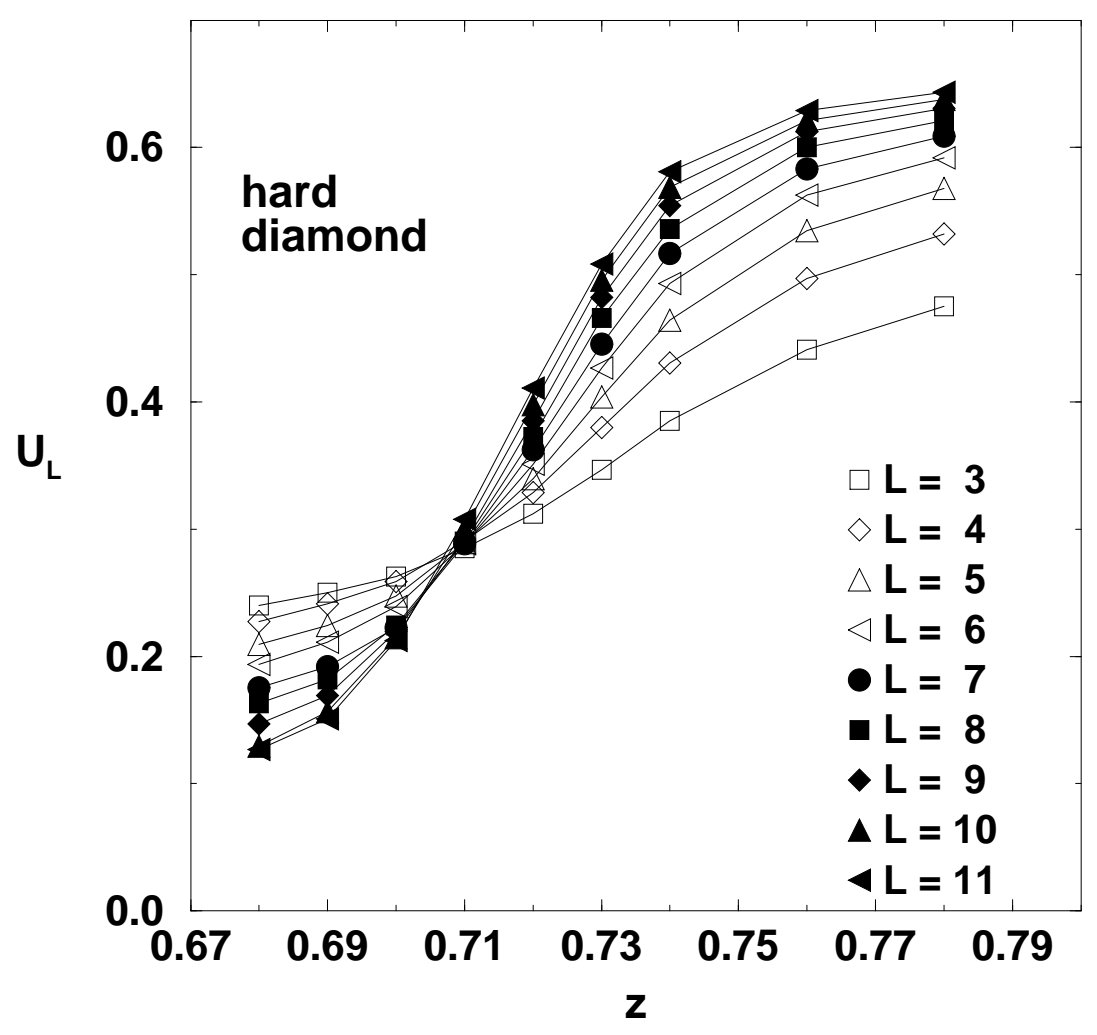

Figure 2 (Nielaba and Lebowitz) 


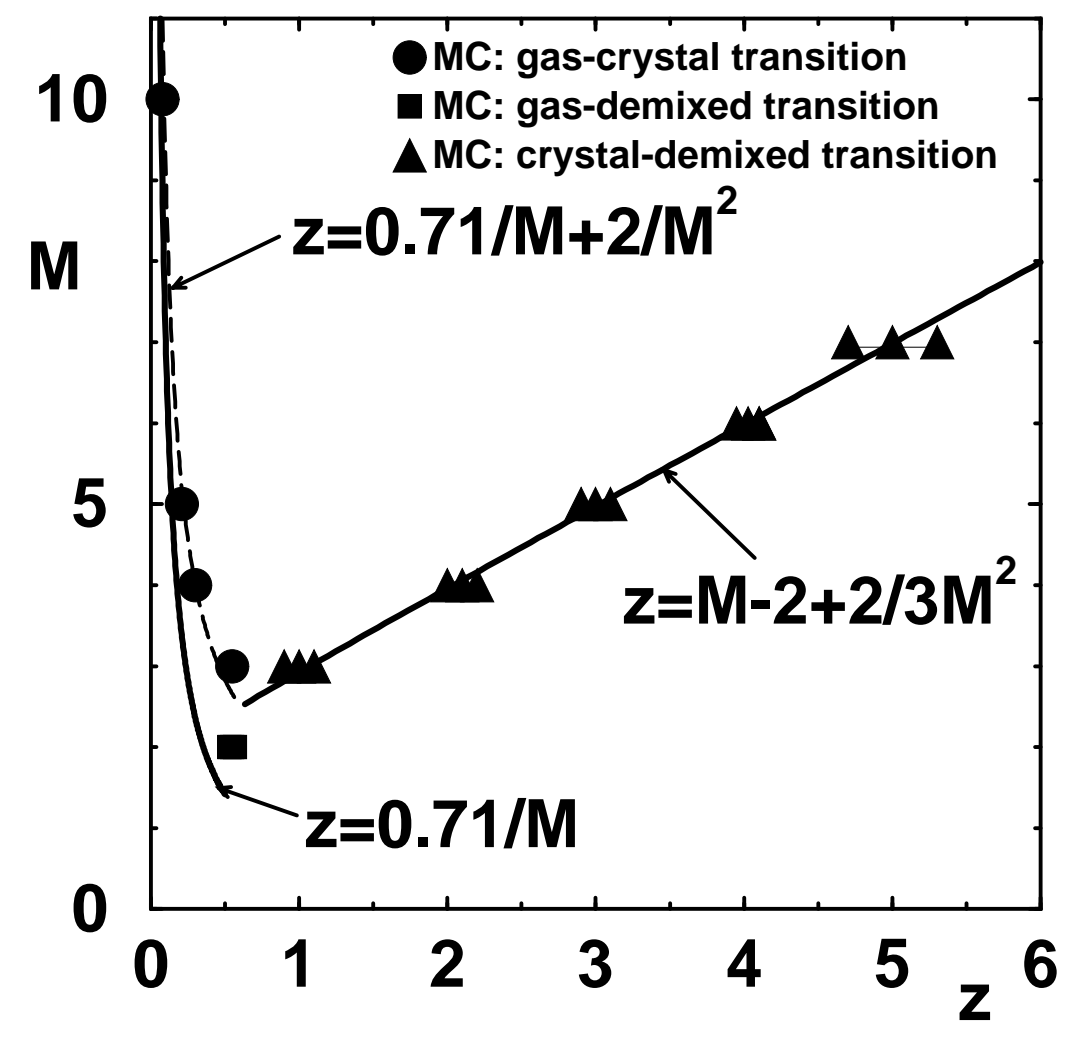

Figure 3 (Nielaba and Lebowitz)

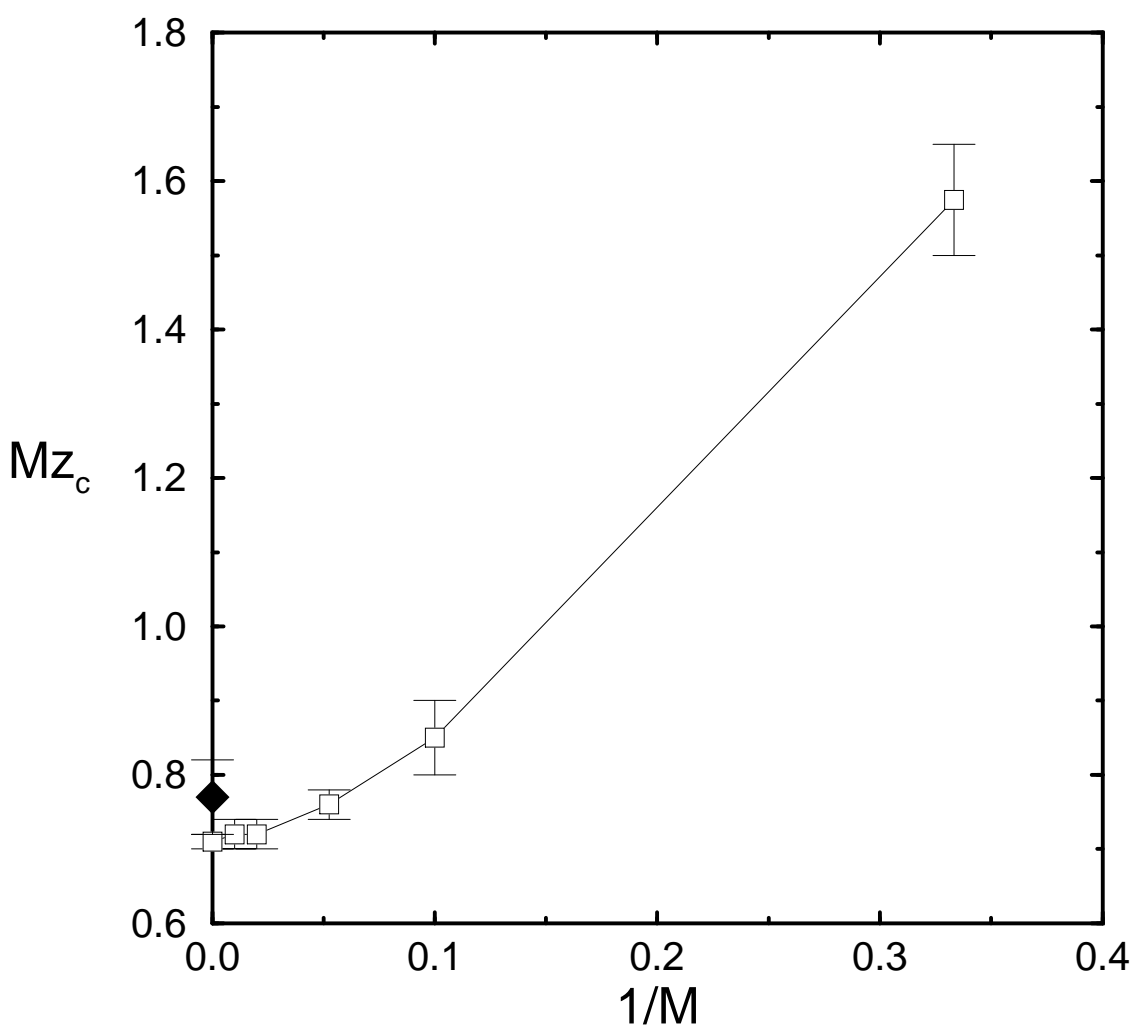

Figure 4 (Nielaba and Lebowitz) 